\title{
Preface: The story of this book
}

This book comes with a story, which we believe is important to know as background to our adventure and endeavour. We had organized an international doctoral course at the Universitat Autònoma de Barcelona, to which Friederike brought ten doctoral students from the University of Siegen and David joined with three doctoral students from Colombia and Saudi Arabia studying at UAB. It was a truly international and interdisciplinary group, with backgrounds in management science, small business studies, accounting, economics, sociology, some with a practice background having recently returned to academia, some with family business experiences, and all now working on their doctoral theses. During the course week, we set out to discuss new research topics in entrepreneurship. But, wherever we went in our discussions, relevance and impact had their place - when we discussed what makes a research topic novel and interesting, when we turned to look at how to best publish from a thesis, when we went to visit an incubator and touched upon how to transfer research results to policy and practice.

Thus, an idea was born: why not discuss the topic from the perspective of early career researchers and a few mid-career researchers? What you hold in your hands in print or look at in electronic form is the result of a huge, year-long joint effort. This book would not have been possible without the many intensive discussions and review rounds that followed the original doctoral seminar, the enthusiasm of the early career researchers and their innovative ideas on how to go about this. A big thank you to all of our contributors! And we also wish to extend our heartfelt thanks to Tatiana Lopez, Anna Müller and Max Paschke for their tremendous assistance in chasing contributors and deadlines, and for the manifold ways they helped us to make this happen.

Friederike Welter and David Urbano 